\title{
Modification of oligosaccharide and short chain fatty acid content of cowpea milk through fermentation with selected mixed starter cultures
}

\author{
${ }^{1,2}$ Kevin O. Aduol, ${ }^{1 *}$ Arnold N. Onyango, ${ }^{1}$ Samuel M. Imathiu
}

\begin{abstract}
${ }^{1}$ School of Food and Nutrition Sciences, Jomo Kenyatta University of Agriculture and Technology, P. O. Box 62000 (00200), Nairobi, Kenya

${ }^{2}$ School of Agriculture and Biotechnology, University of Eldoret, P.O. Box 1125-30100, Eldoret - Kenya,

*Corresponding author, email: arnold.onyango@jkuat.ac.ke
\end{abstract}

https://doi.org/10.37512/400

\begin{abstract}
Cowpea milk was fermented with three mixed starter cultures containing (i) Lactobacillus acidophilus, Bifidobacterium sp, and Streptococcus thermophilus (ABT) (ii) Lactobacillus delbrueckii subsp. bulgaricus and Streptococcus thermophilus (DT) or (iii) Lactobacillus rhamnosus GR-1 and Streptococcus thermophilus (GT). Effects of these cultures on flatulencecausing raffinose family oligosaccharides and the production of postbiotic short chain fatty acids were determined. The oligosaccharides and short chain fatty acids were determined by high performance liquid chromatography and gas chromatography, respectively. The stachyose content of raw cowpea $(1.388 \pm 0.23 \mathrm{~g} / 100 \mathrm{~g})$ was higher than raffinose $(0.221 \pm 0.06 \mathrm{~g} / 100 \mathrm{~g})$, while verbascose was not detected. Cowpea milk fermentation caused $67-100 \%$ reduction in raffinose and $20-70 \%$ reduction in stachyose in a culture-dependent manner. All the cultures produced propionic acid, butyric acid and valeric acid in differing concentrations but only GT and ABT produced isovaleric acid. The product fermented with DT attained $2430 \mathrm{ppm}$ of propionic acid, which was four times and ten times higher than the concentrations produced by the ABT and GT cultures, respectively. In conclusion, fermentation of cowpea milk with the three starter cultures reduced flatulence-causing oligosaccharides and produced postbiotic short chain fatty acids that might promote health regardless of the survival of the microorganisms in the gut.
\end{abstract}

KEY WORDS: Fermentation, Legume milk, Probiotic, Prebiotic

\section{INTRODUCTION}

Non-communicable diseases such as diabetes, cardiovascular diseases and cancers are increasing in prevalence throughout the world, and especially in developing countries (Gowshall and TaylorRobinson, 2018; Somasundaram and Kalupahama, 2016). One of the recommended strategies for reversing this trend is adoption of health-promoting diets (Somasundaram and Kalupahama, 2016). Thus, nutraceuticals, defined as foods or food constituents with health benefits beyond the traditional nutrients, are gaining ground (Schmitt and Ferro, 2018). Probiotic foods are a major class of nutraceuticals; they contain high populations of health-promoting microorganisms (Nagpal et al.,
2012). Originally the definition of probiotics was limited to those microorganisms that when ingested, can survive the harsh conditions in the stomach and exert beneficial effects in the intestines, with the concentration of probiotics required to obtain a clinical effect often being quoted as $\geq 10^{6}$ colony forming units in the small intestine and $\geq 10^{8}$ in the colon (Minelli and Benini, 2008). However, recently the beneficial effects of microorganisms in the oral cavity has also attracted attention (Deogade, 2015). Probiotics induce health benefits by multiple mechanisms including inhibiting the growth of pathogenic microorganisms and reducing the production of inflammatory factors by pathogens (Deogade, 2015). Metabolites produced by probiotics 
contribute to the suppression of pathogens as well as health benefits not associated with pathogen inhibition. For example, short chain fatty acids such as propionic acid and butyric acid have been found to promote apoptosis of colon cancer cells, and to reduce the risk for obesity, insulin resistance, diabetes, hypercholesterolemia, and cardiovascular dysfunctions (Sivaprakasam et al. 2016; Koh et al., 2016; Canfora et al., 2015; Alvaro et al., 2008; Anderson et al., 1999). Such beneficial metabolites are called postbiotics (Cicenia et al., 2014; Klemashevich et al., 2014).

Legumes including cowpeas have a high potential as nutraceuticals (Trinidad, 2010). Cowpea has been suggested to reduce the risk for gastrointestinal diseases, cardiovascular diseases, diabetes and some types of cancers, and this may be due in part to bioactive peptides and phenolic substances (Jayathilake et al., 2018; Awika et al., 2017). However, cowpea consumption is associated with flatulence and other abdominal discomforts in some individuals (Ndubuaku et al., 1989). The raffinose family oligosaccharides including raffinose, stachyose and verbascose are considered to contribute to flatulence (Granitto et al., 2001). On the other hand, these oligosaccharides are also considered to be beneficial as prebiotics which promote the growth of probiotics (Zartl et al., 2018). Legume milks as alternatives to bovine milk constitute a fast-growing segment of functional foods development, fueled by concerns about cow milk allergy, lactose intolerance, hypercholesterolemia, and high calorie diets as well as the presence of health promoting phytochemicals in the legumes (Sethi et al., 2016). Although not as well balanced in amino acids as cow's milk, they may provide a cheap alternative to the less economically empowered, especially where milk supply is insufficient (Sethi et al., 2016).

Fermentation of legumes is an effective way of reducing alpha-galactosidic compounds (Granito et al, 2003). The consumption of fermented beans was found to significantly decrease the flatulence problem by $56 \%$ in women aged 25 to 40 years (Granito et al., 2005). Fermentation also reduces antinutritional factors such as tannins, phytates, alkaloids, hydrogen cyanide, lectins, and oxalates in legumes (Difo et al., 2014). Thus, fermentation of cowpea milk with probiotic lactic acid bacteria is expected to produce a functional food with reduced antinutritional factors, and enhanced health-promoting effects due to probiotics and postbiotics. Here we fermented cowpea milk with three mixed lactic acid bacteria starter cultures and determined the effect of fermentation on the raffinose family oligosaccharides and the production of postbiotic short chain fatty acids. All the cultures contained Streptococcus thermophilus, and, in addition, one of the cultures contained Lactobacillus delbrueckii susb bulgaricus, the second contained Lactobacillus rhamnosus GR-1 strain, and the third contained Lactobacillus acidophilus and Bifidobacterium sp. All these bacteria have previous claims of probiotic effects (Deogade, 2015; Reid, 2017).

\section{MATERIALS AND METHODS}

Cowpeas (white black-eyed variety) were purchased from a local market in Nairobi, Kenya. Three commercial bacterial starter cultures were used for the fermentation of cowpea milk. The cultures containing (i) Lactobacillus acidophilus, Bifidobacterium, Lactobacillus delbrueckii subs. bulgaricus and Streptococcus thermophilus (ABT) and (ii). Lactobacillus delbrueckii subs bulgaricus and Streptococcus thermophilus (DT) were purchased from Prolab, an agent of Chris Hansen, in Nairobi. The culture consisting of Lactobacillus rhamnosus GR-1 strain and S. thermophilus (GT) was obtained from Yoba Foundation. GT, which was in the form of a stable powder was kept at $4 \square \mathrm{C}$, while the other two cu ltures were kept frozen until use, as per manufacturer's instructions.

All chemicals and solvents used in this study were of analytical and chromatographic grade. Oligosaccharide (Stachyose, raffinose and verbascose) standards were bought from Sigma-Aldrich, United Kingdom. Butyric, valeric, and isovaleric acid standards were from Sigma-Aldrich, Germany. Propionic acid standard was bought from Fluka Analytical, Germany. 


\section{Preparation of cowpea milk}

Cowpeas $(300 \mathrm{~g})$ were soaked for 12 hours in 3 liters of distilled water at room temperature (15-25 $\square$ C. After discarding the steeping water, the cowpeas were rinsed with tap water and dehulled manually. Boiling water was used for blending (Von Hotpoint Blender, HB241CW) the cowpeas in the ratio of 1:10 (w/v) for 3 minutes at high speed. The resulting milk was heated to $70 \square \mathrm{C}$ and the temperature held for 20 minutes with frequent stirring to prevent the contents from sticking on the equipment. The milk was cooled to an incubation temperature of $45 \square \mathrm{C}$ and then divided into four portions.

\section{Fermentation of cowpea milk}

Three of the four portions of cowpea milk were inoculated with the starter cultures according to the manufacturer's instructions. No starter culture was added to the control. All the portions were then incubated in an electric incubator at $45^{\circ} \mathrm{C}$ for 14 hours.

\section{Determination of pH}

The $\mathrm{pH}$ of the incubated milk was determined after every 2 hours with a digital $\mathrm{pH}$ meter (HANNA, H18519N) after calibration.

\section{Determination of oligosaccharides}

Oligosaccharides were extracted from cowpea by the method of Borejszo \& Khan (1992). Twenty milliliters of ethanol were added to $5 \mathrm{~g}$ of sample and heated for 1 hour in a reflux apparatus (model SF-6, Sanshin Industrial Co. Ltd). The samples were then concentrated in vacuo and filtered through $0.45 \mu \mathrm{m}$ pore-size membrane syringe filter. Two microliters of the filtrate were injected into High Performance Liquid Chromatography (HPLC) machine model LC-20 AD/T (Shimadzu Corporation, Kyoto Japan) equipped with a degassing unit (DGU- DGU-20A5R) and an autosampler (SIL-20AHT), coupled with refractive index detector model RID-10A and diode array detector (SPD-M20A). Column used was Hypersil GOLD Amino column $(4.6 \times 160 \mathrm{~mm})$. The mobile phase consisted of $65 \%$ acetonitrile and $35 \%$ distilled water and was maintained at a flow rate of $1 \mathrm{~mL} / \mathrm{min}$ isocratically. Identification of the peaks for raffinose, stachyose and verbascose was based on same retention time as the standards, and quantification was based on the use of standard curves obtained under the same HPLC conditions.

\section{Determination of short chain fatty acids}

Extraction of organic acids from cowpea milk samples was carried out according to the procedure by (Shukla et al., 2010). Ten grams of each sample was centrifuged at 10,000 rpm for 30 minutes and then filtered with Whatman filter paper No. 1. Subsequently, $1.2 \mu \mathrm{l}$ of $2 \% \mathrm{H}_{2} \mathrm{SO}_{4}$ was added to $2.1 \mathrm{ml}$ of the filtrate and filtered through $0.45 \mu \mathrm{m}$ pore-size membrane syringe filter. After this, $2 \mu l$ of the filtrate was injected into a Gas Chromatography (GC) machine (Model GC-14B, Shimadzu Corporation, Japan) fitted with a flame ionization detector (FID) and Shimadzu C-R6A Chromatopac and 10\% SUPELCOWAX capillary column $(30 \mathrm{~m} \times 0.53 \mathrm{~mm} \times 0.5 \mu \mathrm{m})$. Carrier gas was nitrogen at a flow rate of $150 \mathrm{~cm}^{3}$ per minute. The column temperature was programmed from $150^{\circ} \mathrm{C}(1 \mathrm{~min})$, to $230^{\circ} \mathrm{C}(2 \mathrm{~min})$ at $5^{\circ} \mathrm{C} / \mathrm{min}$. Injector and detector temperatures were $220^{\circ} \mathrm{C}$ and $240^{\circ} \mathrm{C}$ respectively. Short chain fatty acid (propionate, butyrate, valerate, isovalerate) peaks were identified by comparison with retention times of the respective standards, and standard curves were used for quantification. 


\section{RESULTS AND DISCUSSION}

\section{Change in pH over time during cowpea milk fermentation}

During incubation of cowpea milk (control) or cowpea milk inoculated with the different starter cultures, $\mathrm{pH}$ was monitored over a 14-hour period as an indicator of the progress of fermentation. As illustrated in Figure 1, the control sample exhibited some, albeit minimal decrease in $\mathrm{pH}$, consistent with a small extent of natural fermentation. On the other hand, samples fermented with the three starter cultures underwent a rapid $\mathrm{pH}$ decline from about $\mathrm{pH} 7$ to $\mathrm{pH} 5$ within the first 6 hours, followed by a slower decrease to $\mathrm{pH} 4.5$ (ABT and GT) or 4.8 (DT) between the $6^{\text {th }}$ and $14^{\text {th }}$ hours.

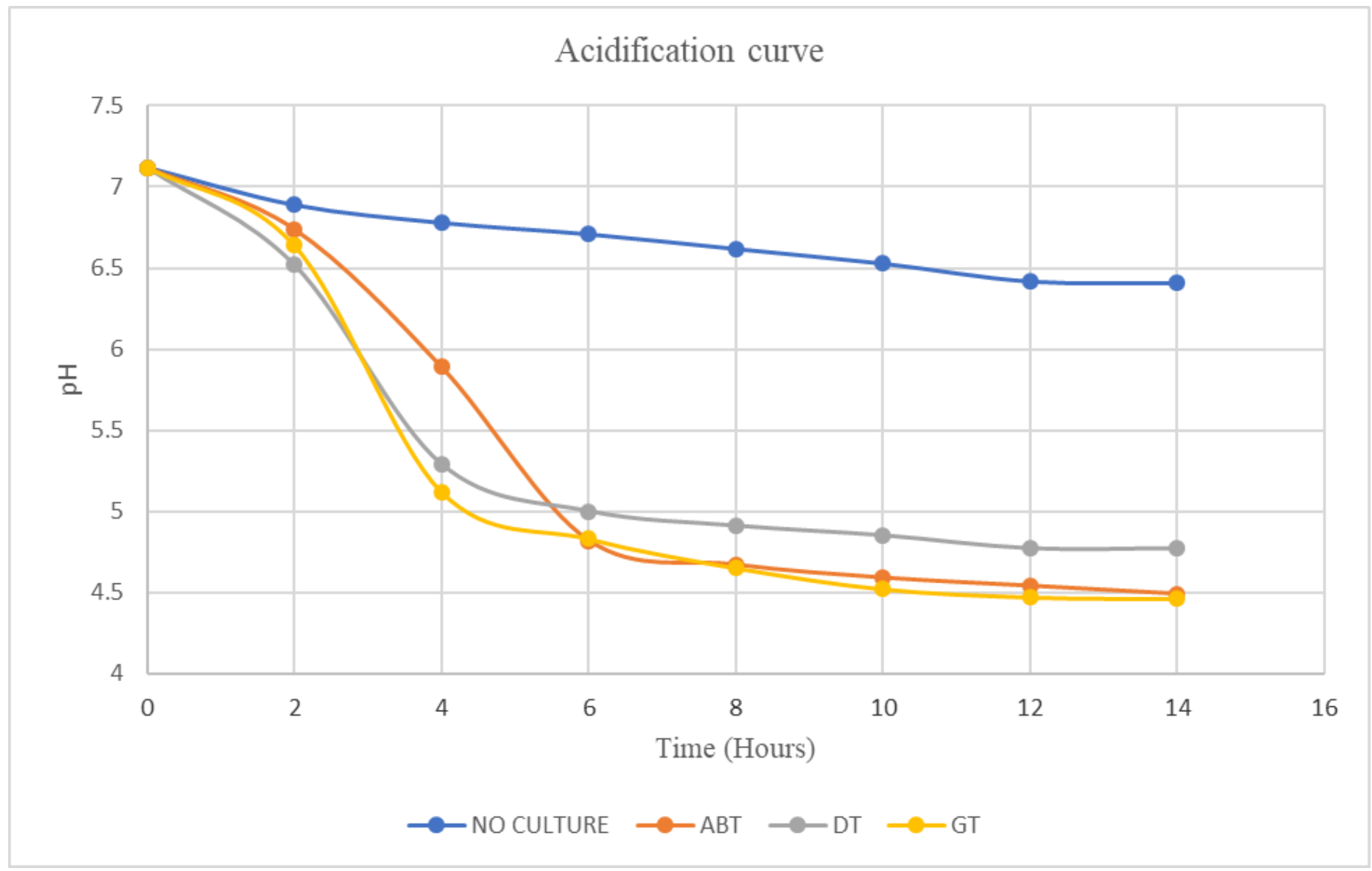

Figure 1. Changes in $\mathrm{pH}$ over time during fermentation of cowpea milk

\section{Effect of fermentation with the different starter cultures on cowpea oligosaccharide content}

Table 1 shows the content of the oligosaccharides stachyose, raffinose and verbascose in raw cowpea flour, unfermented cowpea milk and cowpea milk fermented with the different starter cultures.
Stachyose was detected in all samples, raffinose was detected in all but the ABT and GT-fermented samples, while verbascose was not detected in any of the samples. In all cases, stachyose content was higher than raffinose. 
Table 1: Oligosaccharide concentrations in raw cowpea (RC), unfermented cowpea milk (UM) and cowpea milk fermented with different starter cultures

\begin{tabular}{|c|c|c|c|c|}
\hline \multirow[t]{2}{*}{ Treatment } & \multicolumn{4}{|c|}{ Oligosaccharide concentrations (mg/g) } \\
\hline & Stachyose & Raffinose & Verbascose & Raffinose + Stachyose \\
\hline $\mathbf{R C}$ & $13.9 \pm 0.2^{\mathrm{c}}$ & $2.2 \pm 0.1^{\mathrm{f}}$ & ND & 16.1 \\
\hline UM & $8.8 \pm 0.1^{\mathrm{a}}$ & $0.6 \pm 0.0^{\mathrm{e}}$ & ND & 9.4 \\
\hline ABT & $3.6 \pm 0.0^{\mathrm{b}}$ & $0.0 \pm 0.0$ & ND & 3.6 \\
\hline GT & $7.0 \pm 0.1^{\mathrm{ab}}$ & ND & ND & 7.0 \\
\hline DT & $2.6 \pm 0.0^{\mathrm{bd}}$ & $0.2 \pm 0.0^{\mathrm{e}}$ & ND & 2.8 \\
\hline
\end{tabular}

Values are mean \pm standard deviations. Values with different letter superscript in the same column are significantly different at $(\mathrm{p}<0.05)$ based on Bonferroni tests. ND $=$ Not Detected

Sreerama et al (2012) found higher contents of the three oligosaccharides than presented in Table 1 for raw cowpea, but a similar trend of stachyose $(17.8$ $\mathrm{mg} / \mathrm{g})>$ raffinose $(10.3 \mathrm{mg} / \mathrm{g})>$ verbascose $(3.6 \mathrm{mg} /$ g). Unfermented cowpea milk (UM) had lower content of stachyose and raffinose than the raw cowpea (RC) (Table 1). Since the cowpeas were soaked for 12 hours and dehulled prior to milk extraction, the lower oligosaccharide content in the UM is attributable to their leaching into the soaking water and removal with the hulls. Such oligosaccharide-reducing effect of legume soaking and dehulling has been previously reported (Akinyele et al, 1991; Onyenekwe et al, 2000). Fermentation of cowpea milk further reduced the oligosaccharide contents, and the extent of reduction of each oligosaccharide depended on the culture used (Table 1). The GT culture depleted raffinose but only minimally reduced the stachyose content from 8.8 in unfermented cowpea milk to 7 mg./g (20\% reduction). On the other hand, DT did not deplete raffinose, but reduced stachyose by $70 \%$ reduction. ABT depleted raffinose and reduced stachyose by $59 \%$. In terms of combined stachyose and raffinose reduction, DT was the most effective (70\%), followed by ABT (62\%) and GT $(26 \%)$. Thus, DT and ABT might be more effective in reducing oligosaccharide-dependent flatulence than GT. Nevertheless, because soaking and dehulling also contribute to oligosaccharide reduction, cowpea milk fermented with GT has less than half the oligosaccharides in raw cowpea (Table 1). Liu et al (2006) found that L. rhamnosus strains $6013,6013+\mathrm{DH}_{1}$ and $6013+\mathrm{GH}_{4}$ completely metabolized raffinose in soybean within six hours and strain-dependently reduced the stachyose content by between 50 and $72 \%$. Streptococcus thermophilus was found to be more efficient than Lactobacillus acidophilus in the metabolism of these oligosaccharides and combining either of these with Bifidobacterium increased the oligosaccharide reduction (Wang et al, 2003). Thus, the different combinations of bacteria in the starter cultures used in this study should have contributed to differences in the extent of oligosaccharide reductions. 


\section{Production of post-biotic short chain fatty acids during cowpea fermentation with different starter cultures}

Table 2 shows the effect of cowpea milk fermentation with different starter cultures on the formation of postbiotic short chain fatty acids. Butyric acid, valeric acid and isovaleric acid were not detected in the unfermented, control sample (UM). However, this sample was found to contain a small quantity of propionic acid (50ppm). This can be attributed to natural fermentation, and corresponds to the slight decline in $\mathrm{pH}$ of control sample during the 14-hour incubation (Figure 1). The ABT culture produced small quantities of all the short chain fatty acids except isovaleric acid, with valeric acid being the least produced (20 ppm) and propionic acid the most produced (510 ppm). GT culture produced small quantities of all the fatty acids, ranging from $10 \mathrm{ppm}$ of valeric acid to 220 ppm of propionic acid. DT culture also produced all the fatty acids, and remarkably high levels of propionic acid (2430 ppm), which was significantly different from the concentrations produced by the other cultures. As mentioned above, propionic acid formation occurred in the control sample, which is attributable to natural fermentation, perhaps by propionic acid bacteria. Such bacteria might also have contributed to propionic acid formation in the treatment samples, since lactic acid bacteria such as
Streptococcus thermophilus stimulate the growth of propionic acid bacteria, to different extents (Condon et al., 2001). Nevertheless, in other studies, lactic acid bacteria were found to produce a variety of acids including the short chain fatty acids investigated in the present study (Corsetti et al, 1998; Zalan et al, 2010).

Propionic acid is one of the generally recognized as safe (GRAS) food preservatives (Es et al, 2017). It not only inhibits spoilage fungi and bacteria but also pathogenic ones such as salmonella (Haque et al, 2009) and contributes to reducing the risk of various non-communicable diseases (Sivaprakasam et al. 2016; Koh et al., 2016; Canfora et al., 2015; Alvaro et al., 2008; Anderson et al., 1999). Thus, propionic acid may be an important contributor to potential health benefits of cowpea milk fermented with DT culture.

Unlike propionic acid, the concentration of butyric acid produced by the three starter cultures was not significantly different. DT culture exhibited higher production of valeric acid than the other starter cultures. GT exhibited the highest production of isovaleric acid, although this was not significantly different from DT, the only other culture which produced this acid.

Table 2: Concentration (parts per million) of short-chain organic acids in unfermented cowpea (UM) and cowpea fermented with cultures ABT, GT and DT

\begin{tabular}{|c|c|c|c|c|}
\hline Treatment & Propionic acid & Butyric acid & Valeric acid & Isovaleric acid \\
\hline$\overline{\mathbf{U M}}$ & $50 \pm 6^{\mathrm{a}}$ & ND & $\mathrm{ND}$ & ND \\
\hline ABT & $510 \pm 8^{\mathrm{b}}$ & $40 \pm 1^{\mathrm{a}}$ & $20 \pm 1^{c}$ & ND \\
\hline GT & $220 \pm 17^{\mathrm{c}}$ & $40 \pm 1^{a}$ & $10 \pm 1^{\mathrm{c}}$ & $70 \pm 1^{b}$ \\
\hline DT & $2430 \pm 19^{d}$ & $50 \pm 2^{\mathrm{a}}$ & $60 \pm 1^{d}$ & $40 \pm 1^{b}$ \\
\hline
\end{tabular}

Values are mean \pm standard deviations. Values with different letter superscript in the same column are significantly different at $(\mathrm{p}<0.05)$ based on Bonferroni tests. ND $=$ Not detected 


\section{CONCLUSION AND RECOMMENDATION}

Fermentation of cowpea milk with three different mixed starter cultures led to significant reduction in oligosaccharide content and the formation of postbiotic fatty acids. The extent of reduction of specific oligosaccharides or formation of specific short chain fatty acids depended on the starter cultures. Future studies should be undertaken to determine the potential health benefits of such fermented cowpea products.

\section{Conflict of interest}

The authors declare that there is no conflict of interest.

\section{ACKNOWLEDGEMENT}

This study was supported by the Netherlands Initiative for Capacity Building in Higher Education (NICHE) under the NICHE KEN 189 Project. The fundamental support provided by University of Eldoret, Kenya and the Manager, NICHE KEN 189 Project for this work is highly acknowledged by the authors.

\section{REFERENCES}

Akinyele, I. O. \& Akinlosotu, A. (1991). Effect of soaking, dehulling and fermentation on the oligosaccha rides and nutrient content of cowpeas (Vigna unguiculata). Food Chemistry 41, 43-53.

Alvaro, A., Sola, R., Rosales, R., Ribalta, J., Anguera, A., Masana, L. \& Vallve, J. C. (2008). Gene ex pression analysis of a human enterocyte cell line reveals downregulation of cholesterol biosynthesis in response to short $\square$ chain fatty acids. IUBMB Life 60, 757-764.

Anderson, J. W., B. M. Smith, B. M. \& Washnock, C. S. (1999). Cardiovascular and renal benefits of dry bean and soybean intake. American Journal of Clinical Nutrition 70, 464-474.

Awika, J. M. \& Doudu, K. G. (2017). Bioactive polyphenols and peptides in cowpea (Vignaunguiculata) and their health promoting properties: A review. Journal of Functional Foods 38, 686-697.

Borejszo, Z. B. \& Khan, K. H. (1992). Reduction of flatulence $\square$ causing sugars by high temperature extru sion of pinto bean high starch fractions. Journal of Food Science 57, 771-777.

Canfora, E. E., Jocken, J. W. \& Blaak, E. E. (2015). Short-chain fatty acids in control of body weight and insulin sensitivity. Nature Reviews Endocrinology 11, 577-591.

Cicenia, A., Scirocco, A., Carabotti, M., Pallota, L., Marignani, \& Severi, M. C. (2014). Postbiotic activities of Lactobacilli-derived factors. Journal of Clinical Gastroenterology 48, S18-S22.

Condon, S., Cogan, T.M., Piveteau, P., O'Callaghan, J., \& Lyons, B. (2001). Stimulation of propionic acid bacteria by lactic acid bacteria in cheese, End of Project Report, Teagasc, 2001. https://t-

stor.teagasc.ie/handle/11019/1277

Corsetti, A., Gobetti, M., Rossi, J. \& Daminiani, P. (1998). Antimould activity of sourdough lactic acid bac teria: identification of mixture of organic acids by Lactobacillus sanfrancisco CB1. Applied Microbi ology and Biotechnology 50, 253-256

Deogade, S. C. (2015). Probiotics: contributions to oral and dental health. Oral Health and Dental Manage ment 14, 145-154. Difo, H. V., Onyike, E., Ameh, D. A., Ndidi, U. S. \& Njoku, G. C. (2014). Chemical changes during open and controlled fermentation of cowpea (Vigna unguiculata) flour. International Journal of Food Nutrition and Safety 5, 1-10.

Es, I., Khaneghah, A. M., Hashemi, S. M. B. \& Koubaa, M. (2017). Current advances in biological produc tion of propionic acid. Biotechnology Letters 39, 635-645.

Gowshall, M. \& Taylor-Robinson, S. D. (2018). The increasing prevalence of non-communicable diseases in low-middle income countries: the view from Malawi. International Journal of General Medicine 11, 255-264.

Granito, M., Champ, M., David, A., Bonnet, C. \& Guerra, M. (2001). Identification of gas $\square$ producing com- 
nents in different varieties of Phaseolus vulgaris by in vitro fermentation. Journal of the Science of Food and Agriculture 81, 543-550.

Granito, M., Champ, M., \& Guerra, M. (2003). Fermentation effects on $\alpha \square$ galactosides and fibre in beans. Journal of the Science of Food and Agriculture 83, 1004-1009.

Granito, M., Michel, C., Frias, J., Champ, M. \& Guerra, M. (2005). Fermented Phaseolus vulgaris: acceptabil ity and intestinal effects. European Food Research and Technology 220, 182-186.

Haque, M. N., Chowdhury, R., Islam, K. M. S. \& Akbar, M. A. (2009). Propionic acid is an alternative to anti biotics in poultry diet. Bangladesh Journal of Animal Science 38, 115-122.

Jayathilake, C., Visvanathan, R., Deen, A., Bangamuwage, R., Jayawardana, B. C., Nammi, S. \& Liyanage, R. (2018). Cowpea: an overview on its nutritional facts and health benefits. Journal of the Science of Food and Agriculture 98, 4793-4806

Klemashevich, C., Wu, C., Howsmon, D., Alaniz, R. C., Lee, K. \& Jayaraman, A. (2014). Rational identifica tion of diet-derived postbiotics for improving intestinal microbiota function. Current Opinion in Bio technology 26, 85-90.

Koh, A., Vadder, F. D., Kovatcheva-Datchary, P. \& Backhead, F. (2016). From dietary fiber to host physiolo gy: short-chain fatty acids as key bacterial metabolites. Cell 165, 1332-1345.

Liu, D-M., Li, L., Yang, X-Q, Liang, S-Z. \& Wang, J-S. (2006). Survivability of Lactobacillus rhamnosus dur ing the preparation of soy cheese. Food Technology and Biotechnology 44, 417-422

Minelli, E. B. \& Benini, A. (2008). Relationship between number of bacteria and their probiotic effects. Mi crobial Ecology in Health and Disease 20, 180-183

Nagpal, R., Kumar, A., Kumar, M., Behare, P. V., Jain, S. \& Yadav, H. (2012). Probiotics, their health bene fits and applications for developing healthier foods: a review. FEMS Microbiol Lett. 334, 1-15

Ndubuaku, V. O., Uwaegbute, A. C. \& Nnanyelugo, D. O. (1989). Flatulence and other discomforts associate ed with consumption of cowpea (Vigna unguiculata). Appetite. 1989 Dec;13(3):171-81.

Onyenekwe, P. C., Njoku, G. C. \& Ameh, D. A. (2000). Effect of cowpea (Vigna unguiculata) processing methods on flatus causing oligosaccharides. Nutrition Research 20, 349-358.

Reid, G. (2017). The development of probiotics for women's health. Canadian Journal of Microbiology 63, 269-277.

Schmitt, J. \& Ferro, A. (2013). Nutraceuticals: is there good science behind the hype? British Journal of Clini cal Pharmacology 75, 585-587.

Sethi, S., Tyagi, S. K. \& Anurag, R. K. (2016). Plant-based milk alternatives an emerging segment of function al beverages: a review. Journal of Food Science and Technology 53, 3408-3423.

Shukla, S., Bong, T., Park, H., Kim, M., Koo, I., \& Kim, J. (2010) (Doenjang). Food and Chemical Toxicolo gy, 48(8-9), 2005-2010. https://doi.org/10.1016/j.fct.2010.04.034

Sivaprakasam, S., Prasad, P. D. \& Singh, N. (2016). Benefits of short-chain fatty acids and their receptors in inflammation and carcinogenesis. Pharmacology and Therapeutics 164, 144-151.

Somasundaram, N.P. \& Kalupahana. N. S (2016). Population-based dietary approaches for the prevention of noncommunicable diseases. WHO South-East Asia Journal of Public Health 5, 22-26

Sreerama, Y. N., Sashikala, V. B, Pratape, V. M. \& Singh, V. (2012). Nutrients and antinutrients in cowpea and horse gram flours in comparison to chickpea flour: evaluation of their flour functionality. Food Chemistry 131, 462-468

Trinidad, T. P., Mallillin, A. C., Loyola, A. S. \& Sagum, R. S. (2010). The potential health benefits of legumes as a good source of dietary fibre. British Journal of Nutrition 103, 569-574.

Wang, Y-C., Yu, R_C., Yang, H-Y. \& Chou, C-C. (2003). Sugar and acid contents in soymilk fermented with lactic acid bacteria alone or simultaneously with bifidobacteria. Food Microbiology 20, 333-338.

Zalan, Z., Hudacek, J. Stetina, J., Chumchalova, J. \& Halasz, A. (2010). Production of organic acids by Lacto bacillus strains in three different media. European Food Research and Technology 230, 395-404

Zartl, B., Silberbauer, K., Loeppert, R., Viernstein, H., Praznik, W. \& Mueller, M. (2018). Fermentation of non -digestible raffinose family oligosaccharides and galactomannans by probiotics. Food Funct. 2018 Mar 1;9(3):1638-1646. doi: 10.1039/c7fo01887h. Epub 2018 Feb 21. 